\title{
Hypokalemic myopathy in primary aldosteronism: A case report
}

\author{
CHUIFEN WU ${ }^{1}$, JUN XIN $^{2 *}$, MINGHUA XIN ${ }^{2 *}$, HAI ZOU $^{3}$, LIE JING $^{3}$, CAOYONG ZHU $^{3}$ and WENHUI LEI ${ }^{3}$ \\ ${ }^{1}$ Department of Nephrology, Qingyuan People's Hospital, Lishui, Zhejiang 323000; \\ ${ }^{2}$ Department of Urology, The First Hospital of Quanzhou Affiliated to Fujian Medical University, Quanzhou, Fujian 362000; \\ ${ }^{3}$ Department of Nephrology, Lishui Hospital Affiliated to Zhejiang University, Lishui, Zhejiang 323000, P.R. China
}

Received August 13, 2015; Accepted September 5, 2016

DOI: $10.3892 / \mathrm{etm} .2016 .3864$

\begin{abstract}
Primary aldosteronism (PA) is a rare disorder. The majority of patients with PA present with typical features and are easily diagnosed. This disorder is usually diagnosed with hypokalemia, hypertension or an adrenal mass. However, patients with atypical symptoms may present a challenge for diagnosis and treatment. In the present study, a case of PA is described that presented with hypokalemic myopathy simulating polymyositis. The patient was a 44-year-old woman who presented with weakness and difficulty walking. The patient was initially suspected to have PM and was treated with methylprednisolone. The patient was found to have hypokalemia which persisted despite high-dose supplementation of potassium. Adrenal computed tomography revealed a right adrenal mass. Surgical adrenalectomy was conducted. The final pathological diagnosis was benign adrenocortical adenoma. The serum tests remained normal and the patient's symptoms were resolved during the 8-month follow-up.
\end{abstract}

\section{Introduction}

Primary aldosteronism (PA) is a disorder typically characterized by resistant hypertension, hypokalemia and metabolic alkaosis, associated with suppressed plasma renin activity and excessive aldosterone production (1). It is among the most common causes of secondary hypertension; PA is reported to account for $5-13 \%$ of patients with secondary hypertension (2). Aldosterone-producing adenoma and bilateral adrenal hyperplasia are the two most prevalent causes of primary aldosteronism (3). The majority of patients present with typical features and are easily diagnosed. However, patients with atypical symptoms may present a challenge of diagnosis and treatment. Although many cases with PA do

Correspondence to: Dr Wenhui Lei, Department of Nephrology, Lishui Hospital Affiliated to Zhejiang University, 289 Kuochang Road, Liandou, Zhejiang 323000, P.R. China

E-mail: leiwenhui84@163.com

${ }^{*}$ Contributed equally

Key words: primary aldosteronism, hypokalemic myopathy have hypokalemia ( $>50 \%)$, patients with PA associated with hypokalemic myopathy are rare (1). Kotsaftis et al (4) reported a rare case of hypokalemia-induced myopathy as the first manifestation of primary hyperaldosteronism due to unilateral adrenal hyperplasia. In addition, Goto et al (5) reported a case of PA associated with severe rhabdomyolysis due to profound hypokalemia.

In the present study, a case of PA is described who presented at hospital with prominently hypokalemic myopathy (HM) simulating polymyositis (PM). The patient provided informed consent for the publication of this case report.

\section{Case report}

A 44-year-old Chinese woman visited the emergency department of Lishui Hospital of Zhejiang University (Lishui, China) in July 2013 with weakness in the lower extremity and difficulty walking for 2 days. Serum creatine kinase (CK) was 2,373 IU/1 (normal, 30-135 IU/1) and serum potassium was $1.53 \mathrm{mmol} / \mathrm{l}$ (normal, 3.60-5.00 $\mathrm{mmol} / \mathrm{l}$ ). The patient was admitted for suspected PM. The patient had a history of hypertension for 9 years, with a highest recorded blood pressure of $160 / 100 \mathrm{mmHg}$, and had been treated with antihypertensive agents; captopril and indapamide had been administered in the previous 15 months. The patient's blood pressure was maintained at $130-140 / 80-90 \mathrm{mmHg}$ on admission to the emergency department. Recurrent episodes of limb muscle weakness had been experienced for the previous year, but the patient did not see a doctor. The patient had diarrhea for a number of days prior to admission. Physical examination revealed that her blood pressure was $128 / 78 \mathrm{mmHg}$ and her pulse rate was 68 beats per minute. No rash was observed and the thyroid gland was not enlarged. Respiratory and cardiovascular examinations were normal. Abdominal examination was unremarkable. The liver and spleen were not palpable. Muscle power was grade 3/5 over proximal and grade $4 / 5$ for distal muscle groups in all four limbs. Sensory testing was normal. Knee reflex was diminished and plantar response was downward.

Laboratory investigations revealed abnormally high CK 10,767 IU/1 (normal, 22-430 IU/1), increasing gradually to 17,291 IU/1, potassium $2.11 \mathrm{mmol} / 1$ (after potassium supplement; normal, 3.50-5.60 mmol/1), sodium $139.3 \mathrm{mmol} / \mathrm{l}$, chloride $102.6 \mathrm{mmol} / 1$, magnesium $0.65 \mathrm{mmol} / 1$, calcium $2.35 \mathrm{mmol} / 1$ and $\mathrm{CO}_{2} 23.3 \mathrm{mmol} / 1$. Urinalysis revealed $\mathrm{pH} 7.5$, blood +++ and protein +++ . Complete blood count, 
erythrocyte sedimentation rate, blood urea nitrogen, creatinine, glucose, total protein, albumin and thyroid hormones were normal. Autoantibody profiles included antinuclear antibody, anti-extractable nuclear antigen antibodies, anti-double stranded DNA antibodies, and complement, immunoglobulins and rheumatoid factor were normal. Electrocardiography showed sinus rhythm, flat $\mathrm{T}$ waves in all leads and obvious $\mathrm{U}$ waves. Chest radiographs were normal. B-mode ultrasonography of bilateral kidneys detected no abnormalities. Electromyography (EMG) confirmed myogenic damage.

The patient was treated with a 3-day course of $0.5 \mathrm{~g} /$ day methylprednisolone (Pfizer, Inc., New York, NY, USA) since the presence of PM was suspected. As the possibility of drug-induced hypokalemia, which could be deteriorated by diarrhea, was also considered, the administration of indapamide was discontinued at the time of admission. Treatment was initiated by oral and intravenous supplementation of potassium ( $9 \mathrm{~g} /$ day potassium chloride). After 3 days of treatment, muscle weakness improved markedly and blood testing revealed $\mathrm{CK}$ 7,336 IU/1 and potassium $2.52 \mathrm{mmol} / \mathrm{l}$. This treatment course was not consistent with PM, and the hypokalemia persisted in spite of high dose supplementation of potassium. Considering the presence of concomitant hypertension and hypokalemia, it was agreed that the patient was more likely to have PA which prominently characterized HM rather than PM. Steroid use was then discontinued. Further evaluation revealed elevated urinary potassium excretion (45.2 $\mathrm{mmol} / \mathrm{l})$, suppressed plasma renin activity $(<0.1 \mathrm{ng} / \mathrm{ml} / \mathrm{h}$; normal, $0.1-2.0 \mathrm{ng} / \mathrm{ml} / \mathrm{h})$, excessive aldosterone production $(26.6 \mathrm{ng} / \mathrm{dl}$; normal, $3.6-24.0 \mathrm{ng} / \mathrm{dl})$ and extremely high aldosterone-to-renin ratio (>266 ng/dl per $\mathrm{ng} / \mathrm{ml} / \mathrm{h}$; normal, $<30 \mathrm{ng} / \mathrm{dl}$ per $\mathrm{ng} / \mathrm{ml} / \mathrm{h}$ ). The increase in serum aldosterone concentration was $<30 \%$ after $2 \mathrm{~h}$ of standing in the posture test (before vs. after). Adrenocortical functions were normal. Metanephrine and normetadrenaline were in the normal range.

Contrast enhanced computed tomography scan of bilateral adrenal glands showed a $1.8 \times 1.0-\mathrm{cm}$ adenoma in the right adrenal area (Fig. 1). Following surgical removal of the adenoma, neoplastic tissue was fixed with neutral formalin prior to paraffin embedding. The paraffin blocks were cut into $3-\mu \mathrm{m}$ sections, and stained with hematoxylin and eosin. The pathology report confirmed adrenocortical adenoma (Fig. 2). Following surgery, the patient's symptoms were resolved. The serum tests and blood pressure returned to and remained normal (serum potassium, $4.85 \mathrm{mmol} / \mathrm{l}$; and CK, $93 \mathrm{IU} / \mathrm{l}$ ) without any drugs. The patient reported no recurrence of the symptoms during the 8-month follow-up.

\section{Discussion}

Resistant hypertension and hypokalemia are the most common symptoms of PA. In special cases, severe skeletal muscle injury may be complicated and HM may occur $(2,5,6)$. PA prominently characterized by HM is often misdiagnosed as PM in clinical practice because patients with PA who have severe skeletal muscle injury and significantly increased muscle enzyme levels are very rarely encountered by clinicians (2). PM refers to nonsuppurative inflammation of striated muscle due to an autoimmune cause, which is characterized by symmetric proximal muscle weakness, myalgia, elevated serum muscle enzymes,

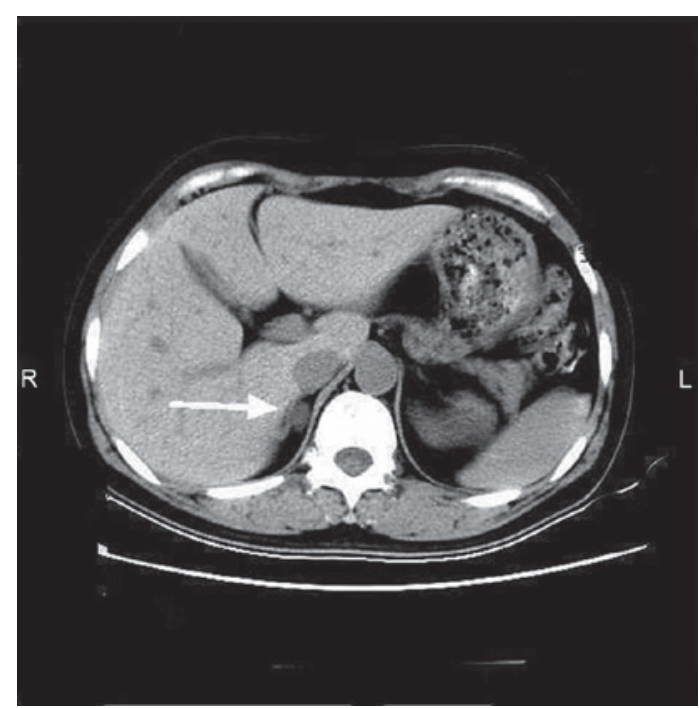

Figure 1. Computed tomography scan of adrenal glands showed a $1.8 \times 1.0-\mathrm{cm}$ adenoma in the right adrenal area (identified by the white arrow). R, right; L, left.

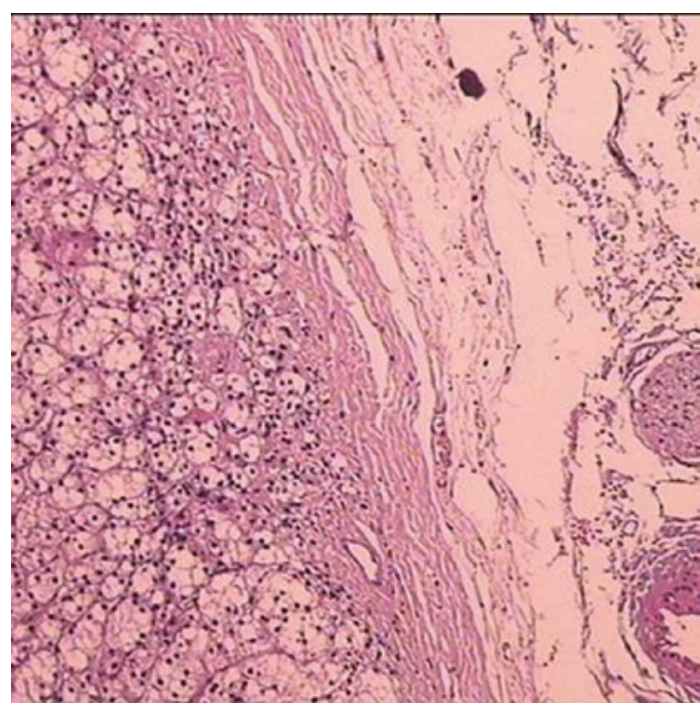

Figure 2. Pathology report confirmed adrenocortical adenoma (elastic Van Gieson stain; magnification, x200).

myogenic damage on EMG, and varying degrees of muscle inflammation and destruction confirmed pathologically (7).

The histopathological findings of HM typically include muscle fiber necrosis, vacuolar changes, and inflammatory cell infiltration and regeneration (6). It is difficult to distinguish between HM and PM histopathologically because of similar histopathological features. Vacuolar changes, which are rarely seen in PM, bear importance in differential diagnosis of these two conditions. However, in a previous study, vacuolar changes were not also observed in patients with HM who underwent muscle biopsy (2). Thus, HM and PM share similar clinical and histological characteristics and cannot be differentiated by determination of muscle enzymes, myoglobins, or EMG findings, or by histopathological evaluation alone. Nevertheless, there are marked differences between HM and PM. HM may be accompanied by profound hypokalemia, while PM is not associated with serum potassium levels, but due to an autoimmune 
cause (8). Patients with HM may achieve rapid and complete improvement following the correction of serum potassium to normal levels and etiology-specific interventions, and are far less likely to experience recurrence, whereas patients with PM may require long-term use of steroids to become stable and are more prone to recurrence (9).

In the present study, the patient had been diagnosed as having essential hypertension since the age of 35 , and had recurrent episodes of limb muscle weakness following treatment with indapamide. Although the record of basic serum potassium level was not acquired, we assume the initiation of the diuretic aggravated the hypokalemia, eventually causing severe HM.

The cause of hypokalemia-induced myopathy in PA is obscure, but a number of reports suggest that the enhanced muscle sodium-potassium pump activity in patients with PA may result in an increase of potassium entry into the cells $(4,6,10)$. The potassium ion is considered to be a major factor mediating the rise of muscle blood flow. When serum potassium level is decreased to below $2.0 \mathrm{mmol} / 1$, a patient with PA may have marked elevation of serum muscle enzymes. In such a case, histological findings include diffuse necrosis and vacuolization of muscle fibers in damaged muscle under light microscopy, and complete dissolution of myofilaments with the disappearance of sarcoplasmic reticulum and T-tubules in the necrotic muscle fibers under electron microscopy. It has been reported that PA associated with hypokalemia-induced rhabdomyolysis or hypokalemic paralysis may be more common in Asian populations (10).

HM and PM are both established causes of myopathy that may be easily confused because of similar presentations. Hypokalemia should be considered in the specific and differential diagnosis of both myopathies. Special care should be taken in the diagnosis of PM. When typical signs of PM and profound hypokalemia are concurrently present, HM should be considered. Concomitant hypertension strongly suggests PA-induced HM. The present case serves to highlight the need for physicians to be aware of the risk of hypokalemia-induced HM among patients, particularly in Asian patients, with PA.

\section{References}

1. Funder JW, Carey RM,Fardella C, Gomez-Sanchez CE, Mantero F, Stowasser M, Young WF Jr and Montori VM; Endocrine Society: Case detection, diagnosis, and treatment of patients with primary aldosteronism: An Endocrine Society clinical practice guideline. J Clin Endocrinol Metab 93: 3266-3281, 2009.

2. Tang YC, Wang SK and Yuan WL: Primary aldosteronism simulating polymyositis. J Rheumatol 38: 1529-1533, 2011.

3. Funder JW, Carey RM, Mantero F, Murad MH, Reincke M, Shibata H, Stowasser M and Young WF Jr: The management of primary aldosteronism: Case detection, diagnosis, and treatment: An Endocrine Society clinical practice guideline J Clin Endocrinol Metabol 101: 1889-1916, 2016.

4. Kotsaftis P, Savopoulos C, Agapakis D, Ntaios G, Tzioufa V, Papadopoulos V, Fahantidis E and Hatzitolios A: Hypokalemia induced myopathy as first manifestation of primary hyperaldosteronism - an elderly patient with unilateral adrenal hyperplasia: A case report. Cases J 2: 6813, 2009.

5. Goto A, Takahashi Y, Kishimoto M, Minowada S, Aibe H, Hasuo K, Kajio H and Noda M: Primary aldosteronism associated with severe rhabdomyolysis due to profound hypokalemia. Intern Med 48: 219-223, 2009.

6. Becker NJ, Hinman M, Giles MN, Kepes JJ and Abdou NI: Polymyositis with hypokalemia: Correction with potassium replacement in the absence of steroids. J Rheumatol 14: 1042-1044, 1987.

7. Sugawara H, Shiraiwa H, Otsuka M and Ueki A: A case of primary aldosteronism presenting hypokalemic myopathy induced by benidipine hydrochloride; a dihydropyridine calcium channel blocker. Rinsho Shinkeigaku 40: 446-451, 2000 (In Japanese).

8. Atluri RB. Inflammatory myopathies. Mo Med 113: 127-130, 2016.

9. Hiraga A, Kamitsukasa I, Kojima K and Kuwabara S. Clinical features and recovery patterns of acquired non-thyrotoxic hypokalemic paralysis. J Neurol Sci 313: 42-45, 2012.

10. Ma JT, Wang C, Lam KS, Yeung RT, Chan FL, Boey J, Cheung PS, Coghlan JP, Scoggins BA and Stockigt JR: Fifty cases of primary hyperaldosteronism in Hong Kong Chinese with a high frequency of periodic paralysis. Evaluation of techniques for tumour localisation. Q J Med 61: 1021-1037, 1986. 